\title{
Spontaneous Symmetry Breaking and Energy Gap Generated by Variables at Infinity ${ }^{\star}$
}

\author{
G. Morchio ${ }^{1}$ and F. Strocchi ${ }^{2}$ \\ 1 Dipartimento di Fisica dell'Università, Pisa, Italy \\ 2 Scuola Normale Superiore, Pisa, and International School for Advanced Studies (ISAS), Trieste, Italy
}

\begin{abstract}
Spontaneous symmetry breaking in the presence of long range instantaneous interactions is studied and the general mechanism underlying it is clarified. A characteristic feature is that the algebraic dynamics does not leave any essentially local algebra stable, i.e. variables at infinity get involved in the time evolution of local variables, so that in each irreducible representation the time evolution fails to be symmetric. For continuous symmetries, the Fourier transform of the vacuum expectation value of charge commutators is related to the energy spectrum at low momenta and a generalized Goldstone theorem is proved which explains the generation of energy gap. This energy gap is further shown to be governed by a "classical dynamics at infinity", equivalently by the group generated by the effective Hamiltonian and the charge. Explicit examples are discussed.
\end{abstract}

\section{General Questions about Spontaneous Symmetry Breaking and Energy Gap}

The phenomenon of spontaneous symmetry breaking appears to be at the basis of several collective effects in many body physics and it plays a crucial role in the unification of elementary particle interactions. Soon after the realization of such a mechanism, considerable interest was devoted to the characterization of the general structures underlying it. The aim was to get both insight on constraints involved in the use of such a mechanism and non-trivial dynamical information on essentially non-linear (collective) effects, without relying on approximations and/or perturbative expansions. The strongest of such characterizations was provided by Goldstone's theorem [1], but it was soon realized that a precise characterization of the hypotheses was crucial for the applicability of the theorem. Actually, from the many examples of many body systems and from elementary particle physics, it became clear that it was important to isolate possible symmetry breaking mechanisms, which allow the evasion of the Goldstone theorem. The

$\star$ Work supported in part by INFN, Sezione di Pisa 
whole problem has been considerably clarified in the last twenty years: the conditions of validity of Goldstone's theorem have been discussed at a high level of rigour and clarity [2] and the "exceptions" to the applicability of the theorem have been essentially identified in the prototypes of the quantum theory of superconductivity and of the Higgs phenomenon.

A folklore explanation for the absence of Goldstone bosons is the presence of long range forces, in the case of non-relativistic systems, and the presence of gauge interactions in the case of elementary particle physics. In our opinion such a cheap explanation does not go to the roots of the problem and it is in some way unsatisfactory, especially for many body systems (it is known that long range correlations do not forbid the applicability of Goldstone's theorem in the case of relativistic quantum field theories). For gauge interactions, a more detailed understanding of the Higgs phenomenon (at least at a perturbative level) has led to identify the Higgs mechanism with some of its more perspicuous features like the presence of gauge fields and the interaction with vector bosons, which "absorb or eat" the would-be Goldstone bosons [3]. In this perspective, however, it is not obvious to draw analogies between the Higgs phenomenon and superconductivity, since the BCS model of superconductivity exhibits a spontaneous breaking of $U(1)$ symmetry with an energy gap, even if the e.m. potential does not appear in the Hamiltonian [4]. This feature appears also in simple field theory models, like the Kibble model [5], and in the linearized Higgs models, once the gauge has been completely fixed in such a way that positivity is preserved.

The above discussion suggests that:

1) The possible analogies between the Higgs phenomenon and superconductivity must be discussed in a more general theoretical setting by identifying general mechanisms, well beyond the folklore explanations,

2) A closely related problem is the characterization of the symmetry breaking aspects of the Higgs phenomenon in the positive gauges; this appears also relevant for non-perturbative controls about the existence of symmetry breaking order parameters (in those gauges) [6].

3) More generally, the identification of a mechanism, which accounts for energy gap generation associated to spontaneous symmetry breaking, without relying on the particular ingredients of the Higgs phenomenon, and therefore with a more general validity, is of interest both for many body theory and for (grand) unified theories of elementary particles. Especially in the latter case, the appearance of Goldstone bosons becomes a serious problem in the construction of realistic theories involving global broken symmetries. This difficulty also appears at the basis of the so-called $U(1)$ problem [7].

4) The phenomenon of energy gap generation associated to a symmetry breaking order parameter may be connected in general with characteristic phenomena of the dynamics of "condensates," as suggested by Kogut-Susskind's mechanism of seizing of the vacuum [8].

A common unifying feature at the basis of the problems 1)-4) is that the corresponding field or many body theories are not formulated in terms of an (essentially) local structure (like a field algebra, or an algebra of localized canonical 
variables) stable under both time evolution and symmetry operations. The evasion of Goldstone's theorem and the possible lack of Goldstone bosons may in fact be traced back to this feature, in great generality. The aim of this work is to provide a general framework for discussing the problems 1)-4), which deals with dynamics and symmetries in the absence of a local structure. This will allow us to characterize the relation between spontaneous symmetry breaking and energy spectrum with a "generalization" of Goldstone's theorem which completely account for the phenomenon of energy gap generation.

\section{Variables at Infinity Generate Energy Gaps}

The generation of an energy gap associated to spontaneous symmetry breaking can be understood in terms of general ideas, which are discussed in this section; the construction of a general framework based on them will be the subject of Sects. 3, 4 .

An essential issue is that long range instantaneous interactions give rise to effects with infinite propagation speed. As a result, if the interaction has sufficiently long range ${ }^{1}$, the time evolution of initially localized variables involves infinitely delocalized variables, essentially "variables at infinity" . Typically, such variables can be represented as ergodic means of localized variables. Equivalently, the general feature is that the boundary conditions not only affect the correlation functions (as in the standard short range case), but they crucially enter into the definition of the equations of motion.

The appearance of variables at infinity in the time evolution of localized variables may give rise to a transfer of charge from localized variables to variables at infinity. As a result, the charge initially localized in a bounded region may leak to infinity in a finite time. This gives rise to oscillations of the total charge measured as the limit of the charge contained in finite volumes $V$, as $V \rightarrow \infty$. The flux of the charge at infinity is therefore non-vanishing. For conserved currents this corresponds to non-vanishing commutators between the flux of the current at infinity $\left(\sim \int d^{3} x \operatorname{div} \vec{j}(x, t)\right)$ and localized variables, and therefore the charge commutators $\int d^{3} x\left\langle\left[j_{0}(x, t), A\right]\right\rangle$ have a non-trivial time dependence. When the charge generates a spontaneously broken symmetry, the associated energy gap is explained precisely by the above time dependence.

The formalization of these ideas leads to the general problem of spontaneous symmetry breaking in the presence of long range (instantaneous) interactions. In the standard case (short range interactions and/or finite propagation speed), the treatment is strongly simplified by the existence of an algebra $\mathscr{A}$ (of local fields or of local dynamical variables) which is stable under time evolution and on which the

\footnotetext{
1 This phenomenon is much more general than the appearance of ergodic limits in the Hamiltonian itself (as in mean field models) and in particular it occurs when the Hamiltonian involves (instaneous) $1 / r$ interactions (see Sect. 5 and ref. [19])

2 They can be identified with variables which can be detected by measurements which can be made outside any finite region [9]. In the following we will in general call variables at infinity variables which commute with any strictly localized variable
} 
symmetry is naturally defined ${ }^{3}$. This property is necessary in order to formulate the symmetry of the time evolution, a condition which plays a crucial role in the Goldstone theorem.

It is worthwhile to remark that the invariance of the (formal) Hamiltonian yields the invariance of the finite volume Hamiltonian $H_{V}$ (in general apart from boundary terms). If the interactions have sufficiently short range (standard case), in the limit $V \rightarrow \infty$ one gets a time evolution which leaves an algebra of (essentially) local dynamical variables stable and is not affected by the boundary terms. Therefore in the standard case the invariance of the formal Hamiltonian yields the invariance of the time evolution of local dynamical variables.

The situation changes drastically in the case of long range interactions which give rise to delocalized boundary terms in the equations of motion so that, in the limit $V \rightarrow \infty$, the time evolution does not leave an essentially local algebra stable. This is what happens i) in general for many body non-relativistic systems with long range potentials, ii) for spin systems with non-integrable interaction potentials, iii) in gauge theories when formulated in terms of non-local variables, which are necessary in order to describe charges obeying a local Gauss law [10].

It then follows that, in the case of long range interactions, the treatment of spontaneous symmetry breaking, from an algebraic point of view, requires an extension of the local algebra to an algebra stable under time evolution, which will in general contain variables at infinity, and the definition of the symmetry on such extended algebra.

The need of defining the symmetry on an algebra containing infinitely delocalized variables gives rise to problems and to new phenomena, if one wants the symmetry to be generated by a local current. In contrast with the standard case, in the limit $R \rightarrow \infty$ the commutator $\left[Q_{R}, A_{t}\right]$ cannot give the total charge associated to the variable $A_{t}$, if the time evolution of $A$ involves variables at infinity, and there is a transfer of charge at infinity. The point is that since $Q_{R}$ commutes with the variables at infinity the conservation of the total charge does not imply that, in the limit $R \rightarrow \infty$, the vacuum expectation value (v.e.v.) of the above commutator is time independent.

In each fixed irreducible representation the picture is the following: the variables at infinity are represented by $c$-numbers and the time dependence of the v.e.v. of the above commutators corresponds to the fact that even if the finite volume Hamiltonians and the finite volume dynamics are symmetric, the infinite volume dynamics of essentially local variables is not symmetric. The v.e.v. of the above commutator represents the time evolution of the localized variable $A$ inside a large bubble of "infinitesimally transformed condensate." Such time evolution is nontrivial essentially because, in contrast with the standard (local) case, the boundary conditions at infinity affect the dynamics inside the bubble of radius $R$, also in the limit of large $R$.

As a result, the charge oscillations discussed above can be described in terms of "oscillations of condensates" which arise because the dynamics (equations of motion) depends on the boundary conditions. Once they are fixed, the corres-

3 For example by using (equal time) canonical (anti-) commutation relations 
ponding dynamics is trivial for condensates (corresponding to the state) with the prescribed boundary condition, but it is non-trivial for condensates corresponding to states with different behaviour at infinity. The frequences of the linearized motion of condensates around the stable point yield the energy spectrum at zero momentum associated to spontaneous symmetry breaking.

\section{Algebraic Dynamics and Symmetries}

An algebraic description of the dynamics ${ }^{4}$ of systems with long range instantaneous interactions meets the following problems. From a constructive point of view, one starts with a net of von Neumann algebras $\mathscr{A}_{V}$ associated to the finite volumes $V$. Space translations $\alpha_{\vec{x}}$ are naturally defined as automorphisms of the "local algebra"

$$
\mathscr{A}_{0} \equiv \bigcup_{V} \mathscr{A}_{V} \text {. }
$$

The finite volume dynamics $\alpha_{V}^{t}$ are defined as one parameter groups of automorphisms of $\mathscr{A}_{0}$, or more generally of its norm closure $\mathscr{A}$. Typically $\alpha_{V}^{t}$ are generated by finite volume Hamiltonians $H_{V}$ affiliated to $\mathscr{A}_{0}$; more generally $\alpha_{V}^{t}$ may describe the dynamics corresponding to an interaction with an infra-red cutoff $V$. The problem is to take the limit $V \rightarrow \infty$.

For interactions with finite propagation speed, for any $A \in \mathscr{A}_{0}, \alpha_{V}^{t}(A)$ becomes independent of $V$, for $V$ large enough, and it defines the time evolution $\alpha^{t}$ as an automorphism of $\mathscr{A}_{0}$. More generally, for interactions with sufficiently short range $\alpha_{V}^{t}(A)$ converges in norm [11] to an automorphism group of $\mathscr{A}$. For spin systems with two-body interaction, the potential must decay faster than $|\vec{x}|^{-3}[11]$.

In the case of long range (instantaneous) interactions, in particular for spin systems with potentials decaying slower than $|\vec{x}|^{-3}$, the finite volume dynamics $\alpha_{V}^{t}$ do not converge in norm and a weaker topology is needed. Physical considerations would suggest that the expectation values $\alpha_{V}^{t}(A)$ converge. The convergence for any state over the algebra $\mathscr{A}$ coincides with the weak convergence with respect to the dual $\mathscr{A}^{\prime}$, and it defines $\alpha^{t}(A)$ as an element of $\mathscr{A}^{\prime \prime}$, the universal von Neumann algebra of $\mathscr{A}$. In all interesting cases, however, the time evolution for large $V$ involves strongly delocalized variables, the expectation values of which converge only if the states are sufficiently regular at infinity ${ }^{5}$.

As a matter of fact both for gauge theories and for many body non-relativistic systems simple physical considerations indicate that the definition of the algebra itself (of dynamical variables) makes implicit reference to a class of states. For gauge theories, non-local field variables correspond to non-local morphisms of the gauge invariant algebra, which describe operations like removing a charge at infinity; this limit is expected to make sense only with reference to a limited class

4 All the mathematical details and proofs will appear in a separate paper, where we also discuss the relation with other approaches [12]

5 A detailed discussion of (physically relevant) models which exhibit such phenomena is deferred to a subsequent paper. Typical examples are the BCS model [4,13], the Kibble model [5] and a large class of mean field spin models (see Sect. 5 and ref. [19]) 
of representations of the gauge invariant algebra. For many body systems the non-relativistic description makes reference to states which involve only nonrelativistic energies. In both cases it is therefore natural to associate to a system an algebra of dynamical variables and a class of state $F$, which are at the basis of the physical interpretation of such an algebra. In this perspective, it is natural to require that $\alpha_{V}^{t}$ converges weakly with respect to the above class of states.

To define an algebraic dynamics in the infinite volume limit we need an algebra $\mathfrak{M}$ and a time evolution $\alpha^{t}$ which is a one parameter group of automorphisms of $\mathfrak{M}$. An important issue is that such structure be uniquely determined by $\alpha_{V}^{t}$ and the local algebra $\mathscr{A}$, so that the extrapolation involved in the infinite volume limit does not require essentially new input with respect to the local structure. A solution of this problem based on the above ideas is given in ref. [I]. The resulting structure is defined by

1) a family $F$ of continuous linear functionals over $\mathscr{A}$ with the following properties: i) $F$ is closed under linear combinations, ii) $F$ is norm closed and separating, i.e. $\phi(A)=0, \forall \phi \in F$, implies $A=0$, iii) $F$ is "stable under local operations" in the sense that if $\phi \in F$, also $\phi_{A B}(\cdot) \equiv \phi(A \cdot B)$, with $A, B \in \mathscr{A}$, belongs to $F$. The positive part $F^{+}$ of $F$ is thus a full folium as in ref. [14]. These states can be taken to be normal states when restricted to the von Neumann algebras $\mathscr{A}_{V}$;

2) the von Neumann algebra $\mathfrak{M}$ constructed as the closure of $\mathscr{A}$ in the weak topology $\tau_{F}$ defined by $F$ on $\mathscr{A}$. As an abstract von Neumann algebra $\mathfrak{M}$ is defined by its predual $F$;

3) a one parameter group $\alpha^{t}$ of ( $\tau_{F}$ continuous) automorphisms of $\mathfrak{M}$, which on $\mathscr{A}$ is the limit of the finite volume dynamics $\alpha_{V}^{t}$ in the ultrastrong topology of $\mathfrak{M}$. As shown in ref. (I), the family $F$ of states can be chosen to be maximal.

This structure can be seen as a generalization of Kadison's definition of dynamical system [15]; the essential difference is however that here $\alpha^{t *}$ is not required to be continuous on $F$ in the weak $*$ topology defined by $\mathscr{A}$, This property is in fact equivalent ([15] and ref. [I]) to the stability of $\mathscr{A}$ under time evolution and its failure plays a crucial role in the explanation of energy gap associated to spontaneous breaking of continuous symmetries.

The above mentioned discontinuity properties of $\alpha^{t *}$ can be seen as the quantum version, for systems with infinite degrees of freedom, of the phenomenon of bifurcation in classical dynamical systems: in fact, on physical grounds neighbourhoods of states are naturally defined in terms of a finite number of measurements of local dynamical variables, i.e. according to the weak * topology, and therefore weak * discontinuity of the time evolution implies that states which give arbitrarily close results of measurements at $t=0$, may yield distant results at time $t$.

The above framework is rich enough to allow the algebraic discussion of spontaneous symmetry breaking in the presence of long range instantaneous interactions.

The first problem is the relation between symmetry and dynamics. Since a symmetry $\beta$ is usually given as an automorphism of $\mathscr{A}$, which is in general not 
stable under time evolution, to give a meaning to the equation

$$
\beta \alpha^{t}=\alpha^{t} \beta
$$

one has to extend $\beta$ from $\mathscr{A}$ to $\mathfrak{M}$.

Furthermore, since in a constructive approach one is given the finite volume dynamics $\alpha_{V}^{t}$, it is important to know when the symmetries of $\alpha_{V}^{t}$ (i.e. symmetries of the finite volume Hamiltonian $H_{V}$ ) are also symmetries of the infinite volume dynamics $\alpha^{t}$ (Eq. (3.1)). As shown in ref. [I], in the framework discussed above, given an automorphism $\beta$ of $\mathscr{A}$ which commutes with $\alpha_{V}^{t}$, the family $F$ can always be chosen to be stable under $\beta^{*}$ and $\beta^{*-1}$, and $\beta$ can be extended to a (uniquely determined) automorphism of $\mathfrak{M}$ which commutes with $\alpha^{t}$.

Similarly, as shown in ref. [I], the covariance of $\alpha_{V}^{t}$ under space translations $\alpha_{\vec{x}}$ implies that $\alpha_{\vec{x}}$ defines an automorphism of $\mathfrak{M}$ which commutes with $\alpha^{t}$.

In the standard discussion of the phenomenon of symmetry breaking an important condition is the approximation of the symmetry $\beta$ by "localized" automorphisms $\beta_{R}$ of the local algebra $\mathscr{A}$,

$$
\beta_{R}(A)=U_{R} A U_{R}^{-1}, \quad A \in \mathscr{A}
$$

with $U_{R}$ in some local algebra. Typically, for one parameter groups $\beta^{\lambda}, \lambda \in \mathbb{R}$,

$$
U_{R}=\exp i Q_{R} \lambda
$$

with the charge $Q_{R}$ affiliated to some $\mathscr{A}_{V}$.

In contrast with the standard case, however, automorphisms $\beta^{\lambda}$ of $\mathfrak{M}$ cannot be approximated on $\mathfrak{M}$ by localized automorphisms $\beta_{R}^{\lambda}$ of the form (3.2) with $U_{R} \in \mathfrak{M}$, (not even in the weak topology), unless $\beta^{\lambda}$ is unbroken in all the representations defined by the states of $F$, as shown in ref. [I].

More generally (ref. $[\mathrm{I}]$ ), when variables at infinity get involved in the time evolution of elements of $\mathscr{A}, \beta^{\lambda}$ cannot be generated by a local charge on an algebra stable under time evolution. This provides a natural mechanism for evading the existence of Goldstone modes in the presence of spontaneous symmetry breaking: essentially the equation

$$
\delta\left(A_{t}\right)=\lim _{R}\left[Q_{R}, A_{t}\right]
$$

does not hold.

Many possible reasons by which Eq. (3.4) may fail have been discussed in the literature. An attempt to trace the failure of Eq. (3.4) back to general properties, when $Q_{R}$ is the integral of a conserved current has been made by Swieca [16], who emphasized the relevance of locality, in the form

$$
\lim _{|\vec{x}| \rightarrow \infty}|\vec{x}|^{n}\left[A_{\vec{x}}, B_{t}\right]=0, \quad n=2, \forall A, B \text { local. }
$$

In fact, in this case, if Eq. (3.4) holds at $t=0$ (as can be checked by using canonical (anti-) commutation relations) and the current at $t=0$ is local, i.e. $j_{0}(f), j_{i}(f)$ are local, then the current conservation yields Eq. (3.4), on a state invariant under time translations, using that $\beta$ commutes with $\alpha^{t}$. This analysis however appears 
more as a weakening of the hypothesis of the Goldstone theorem, rather than an identification of the basic mechanism for the generation of an energy gap. This problem becomes more evident if one takes into account the relation between condition (3.4) and the fall off of the potential (essentially $|\vec{x}|^{3} V(x) \rightarrow 0$ ) given by a semiheuristic argument by Swieca $[16,17]$. The first point is that for sufficiently long range interactions the definition itself of the algebraic dynamics requires special care. For example, for spin systems with two body interactions, if the potential is not integrable (essentially $|\vec{x}|^{3} V(x) \nrightarrow 0$ ), $\alpha_{V}^{t}$ does not converge in norm [11] and the dynamics does not exist in the $C^{*}$-algebraic sense. Furthermore, the relation between the fall off of current commutators and the large distance behaviour of the potential requires a more detailed analysis than that provided by Eq. (3.4). In particular, the integrability of the charge density commutators would by itself suggest $n=3$ in condition (3.4), and by Swieca's argument $|\vec{x}|^{3} V(x) \rightarrow 0$, as $|\vec{x}| \rightarrow \infty$; for spin systems this would imply that $\alpha_{V}^{t}$ converges in norm as $V \rightarrow \infty$, i.e. the standard case of local dynamics. Now, if $|\vec{x}|^{3} V(x) \nrightarrow 0$ as $|\vec{x}| \rightarrow \infty$, the definition of the dynamics as given in Sect. 3 becomes relevant, and furthermore one has to distinguish between the validity of Eq. (3.4) for commutators of $j_{0}$ and of $j_{i}$. If the current is local and conserved, then the property,

$$
\left.\int d^{3} x<\left[j_{0}(x, t), A\right]\right\rangle_{0}=\text { indep. of time, }
$$

is equivalent to

$$
\lim _{|\vec{x}| \rightarrow \infty}|\vec{x}|^{2}\left\langle\left[j_{i}\left(f_{\vec{x}}\right), A\right]\right\rangle_{0}=0 .
$$

However, this condition is not equivalent to Swieca's condition for the potential (namely $|\vec{x}|^{2} V(x) \rightarrow 0$ ), since $j_{i}$ may involve space derivatives of fields. In fact, if $j_{i}$ contains a space derivative of the fields, as happens in most non-relativistic cases, the critical power for the fall off the potential for the failure of the above condition is $V(x) \sim|\vec{x}|^{-1}$ (Coulomb potential). If the current is conserved but $j_{i}$ at $t=0$ is not a local operator, as happens in many spin models, then already a potential decreasing as $|\vec{x}|^{-3}$ may lead to a failure of the above condition.

In a pragmatic point of view, the failure of the Goldstone theorem has been identified with the condition

$$
\lim _{R \rightarrow \infty}\left[\dot{Q}_{R}(t), A\right] \neq 0,
$$

but clearly this does not provide much insight on the dynamical structures which are responsible for it.

The framework discussed above offers a general explanation of the failure of Eq. (3.4), by relating it to general continuity properties of the time evolution of states (see ref. [I]). A simple physical picture may help in visualizing the phenomenon. Consider a state $\phi$ and an automorphism $\beta$ of $\mathscr{A}$ approximated by localized automorphisms $\beta_{R}$ in the sense of Eq. (3.2). Then $\beta_{R}^{*} \phi$ describes a state which essentially coincides with $\phi$ on elements of $\mathscr{A}$ localized outside a sphere of radius $R$, and with $\beta^{*} \phi$ inside the sphere. If the state $\phi$ is characterized by a local order parameter $\phi\left(M_{\vec{x}}\right)=m_{\phi}, M_{\vec{x}} \in \mathscr{A}$, then $\beta_{R}^{*} \phi$ is characterized by the order 
parameter $\phi\left(\beta_{R} M_{\vec{x}}\right)=m(\vec{x})$, with $m(\vec{x}) \simeq m_{\phi}$ outside the sphere of radius $R$ and $m(\vec{x})$ $\simeq m_{\beta^{*} \phi}$ inside. This state can thus be visualized as a "bubble" with order parameter $m_{\beta^{*} \phi}$ inside a medium with order parameter $m_{\phi}$. The time evolution of such a bubble is given by $\alpha^{t *} \beta_{R}^{*} \phi$; the interesting point is the behaviour of such a time evolution in the limit $R \rightarrow \infty$, i.e. when the bubble expands all over the space. In the local case, i.e. when the algebra $\mathscr{A}$ is $\alpha^{t}$ stable, the weak $*$ continuity of $\alpha^{t^{*}}$ implies that the time evolution of very large bubbles approaches the time evolution of the state $\beta^{*} \phi$ with uniform order parameter $m_{\beta^{*} \phi}$. One may also say that the time evolution inside large bubbles is independent of the boundary conditions at infinity. In the non-local case, i.e. when $\mathscr{A}$ is not $\alpha^{t}$ stable, the time evolution inside large bubbles, in the limit $R \rightarrow \infty$, is sensitive to the boundary conditions at infinity; more precisely it depends in general on the value of the order parameters at large distances.

The above mechanism becomes particularly relevant when $\phi$ is invariant under time translations, (typically when $\phi$ is the ground state of a representation of $\mathscr{A}$ with implementable time translations and with positive energy) and the algebraic dynamics is $\beta$ symmetric, $\beta \alpha^{t}=\alpha^{t} \beta$. In this case, if the dynamics is "local," then

$$
w^{*}-\lim _{R \rightarrow \infty} \alpha^{t *} \beta_{R}^{*} \phi=\alpha^{t *} \beta^{*} \phi=\beta^{*} \alpha^{t *} \phi=\beta^{*} \phi,
$$

i.e. the time evolution of large bubbles (as seen by localized variables) approaches a constant, as $R \rightarrow \infty$. In the non-local case, a non-trivial time dependence can persist in the limit of large $R$.

As discussed before, when the algebraic dynamics is non-local, one cannot have an algebra stable under time evolution with symmetries generated by local charges on it, more generally the time evolution of the states, $\alpha^{t^{*}}$, is not continuous with respect to the weak topology defined by algebras on which the symmetry is locally approximated. To deal with this problem it is useful to note that in each factorial representation $\pi$ of $\mathscr{A}$, stable under time evolution, the algebraic dynamics $\alpha^{t}$ gets somewhat reduced to a dynamics in which the variables at infinity become $c$-numbers. In this case one may say that $\pi$ leads to an effective localization of the dynamics since the variables at $\infty$ get somewhat frozen to their (vacuum) expectation value and the non-local variables effectively disappear from the (so reduced) dynamics. It is therefore reasonable to expect that the representation $\pi$ defines a reduced algebraic dynamics $\alpha_{\pi}^{t}$ which leaves stable a "local" algebra, i.e. a subalgebra $\mathscr{A}_{l}$ of $\mathfrak{M}$ which does not contain infinitely delocalized variables. Such effective localization of the dynamics can be formalized in the following way.

Definition 3.1. A factorial representation $\pi$ of the family $F$, stable under $\alpha^{t^{*}}$ leads to an effective localization of the dynamics if there exist a subalgebra $\mathscr{A}_{l} \subset \mathfrak{M}$ with the following properties:

i) $\mathscr{A}_{l}$ is faithfully represented by $\pi$,

ii) $\mathscr{A}_{l}$ is weakly dense in $\mathfrak{M}$,

iii) there exists an automorphism $\alpha_{\pi}^{t}$ of $\mathscr{A}_{l}$, which coincides with $\alpha^{t}$ in the representation $\pi$, namely for any state $\phi \in \pi$

$$
\phi\left(\alpha^{t}(A)\right)=\phi\left(\alpha_{\pi}^{t}(A)\right), \quad \forall A \in \mathscr{A}_{l} .
$$

Since $\mathscr{A}_{l}$ is weakly dense in $\mathfrak{M}, \pi\left(\mathscr{A}_{l}\right)$ is weakly dense in $\pi(\mathfrak{M})$ and therefore the 
center of $\pi\left(\mathscr{A}_{l}\right)$ is contained in the center of $\pi(\mathfrak{M})$, which is trivial because $\pi$ is factorial. Since $\mathscr{A}_{l}$ is faithfully represented by $\pi\left(\mathscr{A}_{l}\right)$, it follows that $\mathscr{A}_{l}$ has a trivial center; thus $\mathscr{A}_{l}$ does not contain any infinitely delocalized variable.

Furthermore, since $\pi$ yields a faithful representation of $\mathscr{A}_{l}$, the automorphism $\alpha_{\pi}^{t}$ is unique, if it exists.

The physical meaning of this structure is that the essential non-local effects of the algebraic dynamics are due to the involvement of the variables at $\infty$; once such variables are frozen to $c$-numbers, as happens with the choice of a factorial representation $\pi$, then one obtains a dynamics which maps a complete set $\mathscr{A}_{l}$ of "essentially local variables" into themselves.

The above algebraic structure offers a convenient mathematical framework for the generalization of the Goldstone theorem: the localization algebra $\mathscr{A}_{l}$ is in fact a natural algebra on which symmetries may be locally approximated (see ref. [I]), and furthermore it is stable under a reduced dynamics, $\alpha_{\pi}^{t}$, which coincides with $\alpha_{t}$ in $\pi$.

The relations between $\alpha^{t}, \alpha_{\pi}^{t}$ and their symmetries are discussed in ref. [I].

Remark. It is worthwhile to remark that the effective dynamics $\alpha_{\pi}^{\mathrm{t}}$ has also a raison d'etre from a point of view based on the correlation functions. According to this philosophy, as in Wightman quantum field theory, the theory is formulated in terms of the (unequal time) correlation functions of a field algebra $\mathscr{A}_{1}$ with trivial centre (essentially localizable fields), defined by a space time translational invariant state $\phi_{0}$. We consider the case in which such correlation functions are constructed by using approximate finite volume dynamics $\alpha_{V}^{t}$, through infinite volume limits like

$$
\lim _{V \rightarrow \infty} \phi_{0}\left(B \alpha_{V}^{t}(A) C\right)=\phi_{0}\left(B A^{t} C\right), \quad A, B, C \in \mathscr{A}_{l}, A^{t} \in \mathscr{A}_{l} .
$$

It is important to stress that even if the approximate (finite volume) dynamics $\alpha_{V}^{t}$ commute with an automorphism $\beta$ of $\mathscr{A}_{l}$, the infinite volume dynamics defined on the (essentially local) field algebra $\mathscr{A}_{l}$ by the correlation functions $\phi\left(B \alpha_{V}^{t}(A) C\right), A, B$, $C \in \mathscr{A}_{l}$, as $V \rightarrow \infty$, need not be symmetric, i.e. one may have

$$
\phi\left(B \beta\left(A^{t}\right) C\right) \neq \phi\left(B(\beta(A))^{t} C\right) .
$$

The point is that in general Eq. (3.8) only guarantees the weak convergence of $\alpha_{V}^{t}(A)$ to $A^{t}$ in the representation $\pi$ defined by $\phi_{0}$, and $\beta$ is continuous with respect to the weak topology defined by $\pi$, if and only if $\beta$ is unbroken in $\pi$, (see Sect. 4); thus in general

$$
\beta\left(w-\lim _{V} \alpha_{V}^{t}(A)\right) \neq w-\lim _{V} \alpha_{V}^{t}(\beta(A)) .
$$

In the local case $\alpha_{V}^{t}(A)$ converges to the infinite volume dynamics in the norm topology, with respect to which $\beta$ is always continuous (since it is an automorphism of $\mathscr{A}_{l}$ ).

The above lack of symmetry of the dynamics appears as a very different phenomenon of symmetry breaking with respect to the conventional one: even if the finite volume Hamiltonians are $\beta$-symmetric, not only the correlation function may fail to be symmetric (as in the conventional case) but the dynamics itself ("the 
equations of motion") of the field algebra may turn out to be asymmetric. From a constructive point of view, the mechanism is that the boundary conditions, which characterize the state, not only affect the correlation functions, but also the dynamics ("the equation of motion") defined on the algebra $\mathscr{A}_{l}$ of essentially localizable fields. A symmetric dynamics can be defined only with reference to an algebra $\mathfrak{M}$ with a non-trivial centre. This phenomenon enforces symmetry breaking: as shown in ref. [I], Sect. 6 , the relation $\beta^{\lambda} \alpha_{\pi}^{t} \neq \alpha_{\pi}^{t} \beta^{\lambda}$ implies that $\beta^{\lambda}$ is broken in $\pi$.

The above features are related to long range effects which show up not only at the level of the correlation functions (as in the standard case) but also at the level of the commutators $\left[H_{V}, A\right], A \in \mathscr{A}_{l}$; for large $V$ such commutators involve largely delocalized variables which converge to different values in different representations, as $V \rightarrow \infty$ (see footnote 5 ).

\section{Spontaneous Symmetry Breaking and Non-Local Dynamics: Energy Gap Generation Through Variables at Infinity}

We are now in position to discuss the phenomenon of spontaneous symmetry breaking with essentially non-local dynamics. In a certain sense, this will provide a generalization of Goldstone's theorem to situations in which the infinitely delocalized variables at infinity will be responsible for energy gap generation associated to spontaneous symmetry breaking.

We recall the framework discussed in the previous sections. A dynamical system is described by a von Neumann algebra $\mathfrak{M}$, with predual $F$, and by an automorphism $\alpha^{t}$ of $\mathfrak{M}$, (time evolution). The space translations are assumed to be automorphisms $\alpha_{\vec{x}}$ of $\mathfrak{M}$ and to commute with $\alpha^{t}$. A symmetry $\beta$ is an automorphism of $\mathfrak{M}$, (therefore weakly continuous). Given a representation $\pi$ of $\mathfrak{M}$ with unique cyclic (pure) state $\phi_{0} \in F$ invariant under space and time translations, if a symmetry $\beta$ commutes with space translations

$$
\beta \alpha_{\vec{x}}=\alpha_{\vec{x}} \beta,
$$

then, by the uniqueness of $\phi_{0}, \beta$ is spontaneously broken in the representation $\pi$ iff there is no unitary operator which describes such a symmetry in $\pi$. Equivalently, $\beta$ is spontaneously broken iff there is an operator $A \in \mathfrak{M}$ such that

$$
\phi_{0}(\beta(A)) \neq \phi_{0}(A) \text {. }
$$

In the following we shall consider continuous one-parameter groups of automorphisms $\beta^{\lambda}, \lambda \in \mathbb{R}$, which commute with space translations.

From the existence of a cyclic vector $\psi_{0}$, representing $\phi_{0}$, it follows that in $\pi$ the space and time translations are described by unitary operators $U(\vec{x}), U(t)$ which are assumed to be strongly continuous in $\vec{x}, t$.

Furthermore, as a result of the discussion of Sect. 3, we shall assume that the representation $\pi$ effectively localizes the dynamics on a subalgebra $\mathscr{A}_{l} \subset \mathfrak{M}$, (Def. 3.1), $\mathscr{A}_{l}$ is stable under space translations and that in the state $\phi_{0}, \beta^{\lambda}$ is 
generated on $\mathscr{A}_{l}$ by local charges: i.e. $\mathscr{A}_{l}$ is stable under $\beta^{\lambda}$ and

$$
\left.\frac{d}{d \lambda} \phi_{0}\left(\beta^{\lambda}(A)\right)\right|_{\lambda=0}=i \lim _{R \rightarrow \infty} \phi_{0}\left(\left[Q_{R}, A\right]\right), \quad A \in \mathscr{A}_{l},
$$

where $Q_{R}$ is the integral of a translationally covariant charge density $j_{0}(\vec{x})$ over a region of size $R$. The charge is assumed to be integrable as a commutator:

$$
J(\vec{x}, t) \equiv i\left\langle\psi_{0},\left[j_{0}(\vec{x}), A^{t}\right] \psi_{0}\right\rangle=i\left\langle\psi_{0},\left[j_{0}(\vec{x},-t), A\right] \psi_{0}\right\rangle
$$

is a finite measure in the $\vec{x}$-variable, i.e. there is a Schwartz seminorm \|\|$_{\mathscr{S}}$ such that for $g(t) \in \mathscr{S}(R), f(\vec{x}) \in \mathscr{S}\left(R^{3}\right)$

$$
|J[f g]|<C\|g\|_{\mathscr{S}} \sup _{\vec{x}}|f(\vec{x})| .
$$

The meaning of Eq. (4.3) is that on $\mathscr{A}_{l}$ the derivative $d \beta^{\lambda} / d \lambda$ can be approximated by local charges, equivalently by the derivatives $d \beta_{R}^{\lambda} / d \lambda$ of unbroken automorphisms $\beta_{R}^{\lambda}$.

By Eqs. (4.2), (4.3) the condition of symmetry breaking in the representation $\pi$ can be written as

$$
\lim _{R \rightarrow \infty} \phi_{0}\left(\left[Q_{R}, A\right]\right) \neq 0, \quad A \in \mathscr{A}_{l} .
$$

The relation between symmetry breaking and energy spectrum is given by the following

Theorem 4.1. Under the above assumptions and the spectral condition ( $d E_{\omega}=0$ for $\omega<0)$, the

$$
\lim _{R \rightarrow \infty}\left\langle\left[j_{0}\left(f_{R}\right), A^{t}\right]\right\rangle_{0} \equiv J(t)
$$

where $A^{t}=U(t) A U(t)^{-1}$, exists in the sense of distributions. Furthermore, for any real test function $f \in \mathscr{S}\left(R^{3}\right)$, with $\int f(\vec{x}) d^{3} x=1$, as a tempered distribution in $\omega$

$$
i\left\langle\psi_{0}, j_{0}(f) d E_{\omega} d E_{k} A \psi_{0}\right\rangle
$$

is a continuous function of $k$ and

$$
i(2 \pi)^{3} \lim _{k \rightarrow 0}\left\langle\psi_{0}, j_{0}(f) d E_{\omega} d E_{k} A \psi_{0}\right\rangle=\widetilde{J}(\omega)
$$

$\left(d E_{\omega}, d E_{k}\right.$ are the spectral measures associated to the space and time translations and $J(\omega)$ is the Fourier transform of $J(t))$.

For the proof and related results see ref. [I].

Remark. It is worthwhile to stress that the relevant condition, which allows us to derive a general connection between the charge commutator and the energy spectrum of excitations at $\vec{k}=0$, without special assumptions on the Fourier transform of charge commutators $[16,17]$, is the condition that the charge, as commutator, is well defined as the integral of a density. Essentially this requires that the commutator $\left\langle\left[j_{0}\left(\vec{x}, A^{t}\right]\right\rangle\right.$ decreases as $|\vec{x}|^{-3-\varepsilon}$. This may appear as a stronger fall off property than that implied by Swieca's condition, Eq. (3.5)),

$$
\lim _{|\vec{x}| \rightarrow \infty}|\vec{x}|^{2}\left[A_{\vec{x}}, B^{t}\right]=0 .
$$


The latter condition is however required both for the charge density and for the current density $\vec{j}(\vec{x})$, and therefore the continuity equation implies that the charge density commutator actually decreases as $|\vec{x}|^{-3-\varepsilon}$. Moreover, Swieca's condition implies that $\left[\partial j_{0}(\vec{x}, t) / \partial t, A\right]$ integrates to zero and therefore it excludes the phenomenon of energy gap generation (see Sects. 3, 4). In the preceding discussion, only the charge density commutator is required to be sufficiently regular as $|\vec{x}| \rightarrow \infty$; no general condition, like Eq. (3.5), is required about the fall off of the generic correlation function, and in fact long range behaviours are allowed. In many examples with long range interactions and energy gap generation associated to spontaneous symmetry breaking, the charge density is integrable, as a commutator.

By Theorem 4.1 the discussion of energy gap associated to spontaneous symmetry breaking is reduced to the computation of $J(t)$. To this purpose since the effective time evolution mapping $\mathscr{A}_{l}$ into $\mathscr{A}_{l}$ is given by $\alpha_{\pi}^{t}, \forall A \in \mathscr{A}_{l}, \alpha_{\pi}^{t}(A) \in \mathscr{A}_{l}$ and on $\mathscr{A}_{l}$ Eq. (4.3) holds, we have

$$
J(t)=i \lim _{R \rightarrow \infty}\left\langle\left[Q_{R}, \alpha_{\pi}^{t}(A)\right]\right\rangle=\left.\frac{d}{d \lambda}\left\langle\beta^{\lambda} \alpha_{\pi}^{t}(A)\right\rangle\right|_{\lambda=0}
$$

It is worthwhile to note that a non-trivial $t$-dependence of $J(t)$ is possible compatibly with $\beta^{\lambda} \alpha^{t}=\alpha^{t} \beta^{\lambda}$, just because it is $\alpha_{\pi}^{t}$ and not $\alpha^{t}$ which appears on the right-hand side of Eq. (4.7). This phenomenon has been discussed in Sect. 3 and explained in the terms of the dependence of the effective dynamics from the boundary condition at infinity.

A non-trivial $t$-dependence of $J(t)$ corresponds to a non-trivial energy spectrum of excitations (Theorem 4.1); the following analysis shows that the time dependence of $J(t)$ can be reduced to the non-trivial dynamics of a set of variables at infinity. To this purpose we need the following definition.

Definition 4.2. In the framework discussed above, given a pure state $\phi_{0}$ invariant under space time translations, giving rise to a representation $\pi$ which effectively localizes the dynamics on $\mathscr{A}_{l}$, we denote by $S$ the minimal set of (pure) states on $\mathscr{A}_{l}$ containing $\phi_{0}$ and stable under $\alpha_{\pi}^{t *}$ and $\beta^{\lambda *}$. We then consider the representation $\Pi_{S}$ of $\mathscr{A}_{l}$ given by the direct sum of all representations defined by states of $S$ and we denote by $Z_{S}$ the centre of $\Pi_{S}\left(\mathscr{A}_{l}\right)$.

If $\mathscr{A}_{l}$ is weakly asymptotically abelian with respect to space translations, in the representation $\pi$, i.e. $\forall A, B \in \mathscr{A}_{l}$.

$$
w-\lim _{|\vec{x}| \rightarrow \infty} \pi\left(\left[A_{\vec{x}}, B\right]\right)=0,
$$

then, since the space translations are implemented by continuous unitary operators, the ergodic limit

$$
w-\lim _{V \rightarrow \infty} \frac{1}{V} \int_{V} d^{3} x \pi\left(A_{\vec{x}}\right)
$$

exists for any $A \in \mathscr{A}_{l}$ and it commutes with $\pi\left(\mathscr{A}_{l}\right)$ [18].

Since the (pure) states of $S$ can be obtained from $\phi_{0}$ by application of $\beta^{\lambda *}, \alpha_{\pi}^{t *}$ and the space translations $\alpha_{\vec{x}}$ commute with both $\beta^{\lambda}$ and $\alpha_{\pi}^{t}$, the ergodic means 
of elements of $\mathscr{A}_{l}$ exist as weak limits in the representation $\Pi_{S}$. In fact any pure state $\psi$ of $\Pi_{S}$ is of the form

$$
\psi=\prod_{i} \beta^{\lambda_{i}^{*}} \alpha_{\pi}^{t_{i}^{*}} \phi, \quad \phi \in \pi
$$

and therefore

$$
\psi\left(\frac{1}{V} \int_{V} \alpha_{\vec{x}}(A) d^{3} x\right)=\phi\left(\frac{1}{V} \int_{V} \prod_{i} \alpha_{\pi}^{t_{i}} \beta^{\lambda_{i}} \alpha_{\vec{x}}(A) d^{3} x\right)=\phi\left(\frac{1}{V} \int_{V} \alpha_{\vec{x}} \prod_{i} \alpha_{\pi}^{t_{i}} \beta^{\lambda_{2}}(A)\right) d^{3} x
$$

converges as $V \rightarrow \infty$.

In the same way one proves that $\mathscr{A}_{l}$ is weakly asymptotically abelian in the representation $\Pi_{S}$, and therefore the ergodic limits of elements of $\mathscr{A}_{l}$ belongs to $Z_{S}$. Actually $Z_{S}$ is generated by such ergodic limits, by the Stone-Weierstrass' theorem.

We are thus led to the following results:

1) $\forall A \in \mathscr{A}_{l}$, the ergodic limit

$$
\lim _{V \rightarrow \infty} \frac{1}{V} \int_{V} \alpha_{\vec{x}}(A) d^{3} x \equiv \lim _{V} A_{V} \equiv A_{\infty}
$$

exists in the weak topology of $\Pi_{S}$ and it belongs to $Z_{S}$.

2) The unique extensions of $\beta^{\lambda}$ and $\alpha_{\pi}^{t}$ to $\Pi_{S}\left(\mathscr{A}_{l}\right)^{11}$ map $Z_{S}$ into $Z_{S}$.

3) Given $A \in \mathscr{A}_{l}$ and $J(t)$ as in Theorem 4.1,

$$
\phi_{0}\left(\beta^{\lambda} \alpha_{\pi}^{t}\left(A_{\infty}\right)\right)=\phi_{0}\left(\beta^{\lambda} \alpha_{\pi}^{t}(A)\right),\left.\quad \frac{d}{d \lambda} \phi_{0}\left(\beta^{\lambda} \alpha_{\pi}^{t}\left(A_{\infty}\right)\right)\right|_{\lambda=0}=J(t) .
$$

Remark. Equation (4.12) reduces the time dependence of $J(t)$, and therefore the phenomenon of energy gap generation, to the time evolution of variables at infinity. Since they form an abelian algebra, stable under such evolution, the problem is reduced to the study of a "classical dynamical system."

Each representation of $\Pi_{S}$ maps such "classical variables" into $c$-numbers and, in particular, it identifies the initial value of the time evolution $\alpha_{\pi}^{t}\left(A_{\infty}\right)$. By definition of $\alpha_{\pi}^{t}$, the dynamical behaviour of such "classical variables" is trivial if the initial values correspond to those of the representation $\pi$, and in general non-trivial otherwise. In particular, a non-trivial time evolution may arise starting from initial values corresponding to $\beta^{\lambda *} \phi_{0}$, when $\beta^{\lambda}$ and $\alpha_{\pi}^{t}$ do not commute. As discussed in Sect. 3, this happens when the effective dynamics $\alpha_{\pi}^{t}$ depends on the boundary conditions which characterize the representation $\pi$.

Equation (4.12) shows that the low momentum energy spectrum in the representation $\pi$ is determined by the time evolution of the ergodic limit $A_{\infty}$, when the initial value is infinitesimally close to that of the representation $\pi$, in the direction of the symmetry transformation $\beta^{\lambda *}$.

The above classical dynamical problem can be further simplified under general assumptions, by which only a finite number of "degrees of freedom at infinity" get involved. A general case in which this happens is when

a) given $A_{1} \in \mathscr{A}_{l}$, there is a $C^{*}$-subalgebra $R$ of $Z_{S}$ which contains $A_{1, \infty}$, which is 
stable under $\alpha_{\pi}^{t}$ and is generated by a finite number of hermitian ergodic limits $z_{i}=\lim _{V \rightarrow \infty} A_{i, V}, i=1, \ldots, n, A_{i} \in \mathscr{A}_{l}$.

It then follows that

$$
\alpha_{\pi}^{t}\left(z_{i}\right)=F_{i}^{t}\left(z_{1}, \ldots z_{n}\right),
$$

with $F_{i}^{t}$ norm limits of polynomials of the $A_{i}$ 's. $F_{i}^{t}$ are then continuous functions. In the following we assume that $F_{i}^{t}$ are differentiable.

Theorem 4.2. Under the above conditions the linearization of the dynamics $z_{i} \rightarrow$ $F_{i}^{t}\left(z_{1}, \ldots, z_{n}\right)$ around the stable point $\bar{z}_{i}=\phi_{0}\left(A_{i}\right)$ gives rise to a motion of the form

$$
x_{i}^{t}=\sum_{k} P_{k}^{i}(t) \exp \left(i \omega_{k} t\right)
$$

with $P_{k}^{i}(t)$ polynomials and $\omega_{k}$ real; the energy spectrum at zero momentum of the two point function involving $A_{i}$, Eqs. (4.5), (4.6), coincides with the set of frequencies $\omega_{k}$ which enter in the linearized motion of $z_{i}$.

Proof. By Eq. (4.13), since $\beta^{\lambda}$ is an automorphism of $Z_{S}$ and therefore it is norm continuous, we have

$$
\beta^{\lambda} \alpha_{\pi}^{t}\left(z_{i}\right)=\beta^{\lambda} F_{i}^{t}\left(z_{1}, \ldots, z_{n}\right)=F_{i}^{t}\left(\beta^{\lambda}\left(z_{1}\right), \ldots, \beta^{\lambda}\left(z_{n}\right)\right) .
$$

Moreover, since $Z_{S}$ is abelian and $\phi_{0}$ is a pure state,

$$
\phi_{0}\left(F_{i}^{t}\left(z_{1}, \ldots, z_{n}\right)\right)=F_{i}^{t}\left(\phi_{0}\left(z_{1}\right), \ldots, \phi_{0}\left(z_{n}\right)\right) .
$$

Now, by Eq. (4.12), $\phi_{0}\left(\beta^{\lambda}\left(z_{i}\right)\right)=\phi_{0}\left(\beta^{\lambda}\left(A_{i}\right)\right)$ which is differentiable in $\lambda$ by condition (4.3); since $F_{i}^{t}$ is differentiable we have

$$
\left.\frac{d}{d \lambda} \phi_{0}\left(\beta^{\lambda} \alpha_{\pi}^{t}\left(z_{i}\right)\right)\right|_{\lambda=0}=\left.\sum_{k} F_{i, k}^{t}\left(\phi_{0}\left(z_{1}\right), \ldots, \phi_{0}\left(z_{n}\right)\right) \frac{d}{d \lambda} \phi_{0}\left(\beta^{\lambda}\left(z_{k}\right)\right)\right|_{\lambda=0} .
$$

With obvious notations, the above equation can be written in the form

$$
x_{i}^{t}=F_{i, k}^{t}\left(\bar{z}_{1}, \ldots, \bar{z}_{n}\right) x_{k}^{0} .
$$

We now show that $F_{i, k}^{t}$ is a polynomially bounded abelian group of real matrices. In fact by Eq. (4.13) and the group properties of $\alpha_{\pi}^{t}$, we have

$$
F_{i}^{t+s}\left(z_{1}, \ldots, z_{n}\right)=\alpha_{\pi}^{t+s}\left(z_{i}\right)=\alpha_{\pi}^{t}\left(\alpha_{\pi}^{s}\left(z_{i}\right)\right)=\alpha_{\pi}^{t} F_{i}^{s}\left(z_{1}, \ldots, z_{n}\right)=F_{i}^{s}\left(\alpha_{\pi}^{t}\left(z_{1}\right), \ldots, \alpha_{\pi}^{t}\left(z_{n}\right)\right),
$$

and therefore

$$
F_{i, k}^{t+s}\left(z_{1}, \ldots, z_{n}\right)=F_{i, j}^{s}\left(\alpha_{\pi}^{t}\left(z_{1}\right), \ldots, \alpha_{\pi}^{t}\left(z_{n}\right)\right) F_{j, k}^{t}\left(z_{1}, \ldots, z_{n}\right) .
$$

By taking the expectation value on the pure (time invariant) state $\phi_{0}$, the above equation gives

$$
F_{i, k}^{t+s}\left(\bar{z}_{1}, \ldots, \bar{z}_{n}\right)=F_{i, j}^{s}\left(\bar{z}_{1}, \ldots, \bar{z}_{n}\right) F_{j, k}^{t}\left(\bar{z}_{1}, \ldots, \bar{z}_{n}\right) .
$$

Finally, since $x_{i}^{t}$ is polynomially bounded in $t$ by Eq. (4.12), (see Theorem 4.1), so are the matrices $F_{i, k}^{t}$. They can therefore be written as $(\exp (K t))_{i k}$ with $K$ a real matrix; the spectrum of $K$ is purely imaginary since $F_{i, k}^{t}$ are polynomially bounded and therefore also symmetric with respect to the origin $(\operatorname{spect} K=(\operatorname{spect} \bar{K}))$. If 
$F_{i, k}^{t}$ are uniformly bounded in $t$, the spectrum of $K$ has no algebraic multiplicity and the linearized motion (4.16) is multiperiodic, with frequencies given by the eigenvalues of $K$. In the general case the spectrum of $K$ has algebraic multiplicities and the corresponding motion (4.16) is described by products of polynomials in $t$ and (periodic) exponentials. The connection with the energy spectrum at zero momentum follows from Theorem 4.1.

Clearly, the same conclusions of the above theorem are obtained if condition $\alpha$ ) is weakened to require the reduction to a finite number of degrees of freedom only in the neighbourhood of the stable point $\bar{z}_{i}$ :

$\left.\alpha^{\prime}\right)$ given $A_{1} \in \mathscr{A}_{l}$, there is a finite number $n$ of hermitian ergodic limits $z_{i}$ such that, for $|\lambda|$ small,

with

$$
\phi_{0}\left(\beta^{\lambda} \alpha_{\pi}^{t}\left(z_{i}\right)\right)=F_{i}^{t}\left(\phi_{0}\left(\beta^{\lambda}\left(z_{1}\right)\right), \ldots, \phi_{0}\left(\beta^{\lambda}\left(z_{n}\right)\right)\right)+R_{i}^{\lambda, t}
$$

$$
\left\|R_{i}^{\lambda, t}\right\|<C^{t} \lambda^{1+\varepsilon}
$$

and $F_{i}^{t}$ a differentiable function.

A reduction to a finite dimensional classical problem is also obtained if only a finite number of symmetries are generated by $\beta^{\lambda}$ and $\alpha_{\pi}^{t}$, more precisely if

$\beta$ ) the automorphisms $\beta_{(t)}^{\lambda}$ of $\mathscr{A}_{l}$, defined by

$$
\beta_{(t)}^{\lambda} \equiv \alpha_{\pi}^{-1} \beta^{\lambda} \alpha_{\pi}^{t}
$$

are generated on $\mathscr{A}_{l}$, in the state $\phi_{0}$, by a finite number of independent (local) charges $Q_{R}^{i}$, i.e. $\forall A \in \mathscr{A}_{l}$

$$
\left.\frac{d}{d \lambda} \phi_{0}\left(\beta_{(t)}^{\lambda}(A)\right)\right|_{\lambda=0}=i \sum_{i=1}^{n} a_{i}(t) \lim _{R} \phi_{0}\left(\left[Q_{R}^{i}, A\right]\right),
$$

with $a_{i}(t)$ independent of $A$.

Since $\mathscr{A}_{l}$ is stable under $\alpha_{\pi}^{t}$, for any fixed $t \beta_{(t)}^{\lambda}$ is generated by $Q_{R}(t)=\alpha_{\pi}^{-t} Q_{R}$ and therefore Eq. (4.18) states that only a finite number of charges $Q_{R}(t), t \in \mathbb{R}$ are independent. For the following it is convenient to choose the charges $Q_{R}^{i}$ as $Q_{R}^{i}=Q_{R}\left(t_{i}\right)$, for suitable times $t_{1}, \ldots, t_{n}$.

Equation (4.18) can then be written as

$$
\begin{aligned}
\frac{d}{d \lambda} \phi_{0}\left(\beta_{t}^{\lambda}(A)\right)_{\lambda=0} & =i \lim _{R \rightarrow \infty} \phi_{0}\left(\left[Q_{R}(t), A\right]\right)=\sum_{i} c_{i}(t) i \lim _{R \rightarrow \infty} \phi_{0}\left(\left[Q_{R}\left(t_{i}\right), A\right]\right) \\
& \left.\equiv \sum_{i} c_{i}(t) \frac{d}{d \lambda} \phi_{0}\left(\beta_{i}^{\lambda}(A)\right)\right|_{\lambda=0}
\end{aligned}
$$

and one has

$$
\alpha_{\pi}^{-t} \beta_{i}^{\lambda} \alpha_{\pi}^{t} \equiv \beta_{i}^{\lambda}(t)=\beta_{t+t_{i}}^{\lambda}
$$

We then have

Theorem 4.3. Under the same assumptions as in Theorem 4.1 if condition $\beta$ ) holds then

$$
\frac{d}{d \lambda} \phi_{0}\left(\beta_{i}^{\lambda}(t)(A)\right)_{\lambda=0}=\sum_{i} c_{i k}(t) \frac{d}{d \lambda} \phi_{0}\left(\beta_{k}^{\lambda}(A)\right)_{\lambda=0},
$$


where $c_{i k}(t)$ is a one-parameter group of real matrices, polynomially bounded in $t$; the spectrum of its generator coincides with the spectrum of $J(\omega)$, and therefore with the energy spectrum at zero momentum, in the sense of Theorem 4.1, when A varies over $\mathscr{A}_{l}$.

Proof. Equation (4.21) follows from (4.19), (4.20) with

$$
c_{i k}(t) \equiv c_{k}\left(t+t_{i}\right) \text {. }
$$

Equation (4.21) with hermitian $A$ shows that $c_{i k}(t)$ are real. Moreover, we have

$$
\begin{aligned}
\sum_{k} c_{i k}(t+s) \frac{d}{d \lambda} \phi_{0}\left(\beta_{k}^{\lambda}(A)\right)_{\lambda=0} & =\frac{d}{d \lambda} \phi_{0}\left(\beta_{i}^{\lambda}(t+s)(A)\right)_{\lambda=0}=\sum_{j} c_{i j}(s) \frac{d}{d \lambda} \phi_{0}\left(\beta_{j}^{\lambda}\left(\alpha_{\pi}^{t}(A)\right)\right)_{\lambda=0} \\
& =\sum_{j, k} c_{i j}(s) c_{j k}(t) \frac{d}{d \lambda} \phi_{0}\left(\beta_{k}^{\lambda}(A)\right)_{\lambda=0},
\end{aligned}
$$

and therefore $c_{i k}(t)$ is a one-parameter group of real matrices. The proof then proceeds as in Theorem 4.2.

Condition $\beta$ ) can be recovered as a condition on the linearized dynamics of $Z_{S}$ around the stable point defined by the state $\phi_{0}$. In fact, condition $\beta$ ) is implied by the following:

$\left.\beta^{\prime}\right)$ the (finite) products of automorphisms $\beta_{(t)}^{\lambda}, \lambda, t \in \mathbb{R}$, form a finite dimensional Lie group $G$ of automorphisms $\beta(g), g \in G$, of $Z_{S}$.

Given $z \in Z_{S}$, with $z$ the ergodic mean of $A \in \mathscr{A}_{l}$ one can choose a basis $L_{i}$ in the Lie algebra of $G$ such that

$$
\beta\left(\exp \lambda L_{i}\right)(z)=\beta_{\left(t_{i}\right)}^{\lambda}(z) \equiv \beta_{i}^{\lambda}(z) .
$$

Moreover, by Eq. (4.12), $\phi_{0}\left(\beta_{(t)}^{\lambda}(z)\right)=\phi_{0}\left(\beta_{(t)}^{\lambda}(A)\right)$ and the latter is differentiable in $\lambda$ at $\lambda=0$, by condition (4.3); therefore

$$
\begin{aligned}
\frac{d}{d \lambda} \phi_{0}\left(\beta_{i}^{\lambda}(t)(z)\right)_{\lambda=0} & =\frac{d}{d \lambda} \phi_{0}\left(\beta^{\lambda}\left(t_{i}+t\right)(z)\right)_{\lambda=0}=\frac{d}{d \lambda} \phi_{0}\left(\beta\left(\exp \lambda \sum_{j} c_{i j}(t) L_{j}\right)(z)\right)_{\lambda=0} \\
& =\sum_{j} c_{i j}(t) \frac{d}{d \lambda} \phi_{0}\left(\beta\left(\exp \lambda L_{j}\right)(z)\right)_{\lambda=0} \\
& =\sum_{j} c_{i j}(t) \frac{d}{d \lambda} \phi_{0}\left(\beta_{j}^{\lambda}(z)\right)_{\lambda=0}
\end{aligned}
$$

Clearly by Eq. (4.12) the above equation holds when $z$ is replaced by $A$ and in such a way one recovers Eq. (4.18).

It is worthwhile to remark that as a state on $\mathscr{A}_{l}$, the state $\beta_{(t)}^{\lambda^{*}} \phi_{0}$ is completely determined (within $S$ ) by its values on $Z_{S}$ and in this way one may define $\beta(g)^{*}$ on $\phi_{0}$ :

$$
\beta(g)^{*} \phi_{0}(\dot{z}) \equiv \phi_{0}(\beta(g)(z))=\phi_{0}\left(\beta_{(t)}^{\lambda}(z)\right)=\beta_{(t)}^{\lambda^{*}} \phi_{0}(z)
$$

Condition $\beta^{\prime}$ ) then implies that the (finite) products of $\beta_{(t)}^{*}$ form a finite dimensional Lie group $\beta(g)^{*}, g \in G$, on $\phi_{0}$. The translation invariance of $\phi_{0}$ may be crucial for the validity of this property. Moreover since $\phi_{0}$ is $\alpha_{\pi}^{t}$ invariant and $S$ is minimal, it also follows that $S=\left\{\beta^{*}(g) \phi_{0}, g \in G\right\}$. 
Remark. The Conditions $\alpha$ ), $\alpha^{\prime}$ ), $\beta$ ), $\beta^{\prime}$ ) imply that the energy spectrum at zero momentum (see Theorem 4.1) consists of discrete points $\omega_{i}$ (excitations with infinite lifetime in the limit $k \rightarrow 0$ ). If $J(t)$ is uniformly bounded, the minimal number of degrees of freedom which enter in the conditions $\alpha$ ) $\alpha^{\prime}$ ), or the minimal number of charges for which Eq. (4.18) holds for fixed $A \in \mathscr{A}_{l}$, is even if and only if $\omega_{i} \neq 0$ for all $i$ 's, i.e. iff one has an energy gap associated to the spontaneous breaking of $\beta^{\lambda}$. In general, given the generator $Q_{R}$ of $B^{\lambda}$ and the generator $Q_{R}^{0}$ of an automorphism $\alpha_{0}^{\lambda}$ which commutes with $\alpha_{\pi}^{t}$, one can always define a new group of automorphism $\gamma^{\lambda}$ with generator $Q_{R}+Q_{R}^{0}$. Therefore the appearance of the point $\omega=0$ cannot be ruled out by the time dependence of $\beta_{(t)}^{\lambda}$. It is clear however that one can get rid of the charge $Q_{R}^{0}$ by a suitable choice of the operator $A$. This separation can be done in general if condition $\beta$ ) holds (ref. [I]).

Remark. Theorems 4.2, 4.3 show that only discrete excitations contribute at zero momentum to the vacuum expectation value of $\left[Q_{R}, A\right]$ if only a finite number of charges $Q_{R}(t), t \in \mathbb{R}$, are independent, or if only a finite number of "condensates" get involved in the linearized (classical) motion of the "condensate" $A_{\infty}$. The converse is also true: if the energy spectrum at $\vec{k}=0$ is discrete, then by a suitable smearing in time of $A_{t}$ one can obtain that no more than two degrees of freedom, with frequencies $\omega$ and $-\omega$, are involved in the dynamics of the condensates or of the commutator $\left\langle\left[Q_{R}(t), A\right]\right\rangle_{0}$ for fixed $A$.

Apart from simple models, the determination of the function $F_{i}^{t}$ in Eq. (4.13), or of the matrices $c_{i k}(t)$ in Eq. (4.21), is in general a non-trivial "dynamical" problem. To attack this problem one may perform a sort of Hartree-Fock approximation on the equation of motion of the variable $A$. Typically, in a given representation the time derivative of a field variable $A$ is expressed in terms of a set of field variables $A_{1}, \ldots, A_{n}$

$$
\dot{A}=F\left(A_{1}, \ldots, A_{n}\right)
$$

and the first approximation is to take

$$
\left(F\left(A_{1}, \ldots, A_{n}\right)\right)_{\infty}=F\left(A_{1 \infty}, \ldots, A_{n \infty}\right) .
$$

Such approximation turns out to be exact for linear models. More generally the above approximation may be taken as a starting point for setting up an iterative procedure in terms of truncated correlation functions. In this way one may get approximate determinations of the energy spectrum at zero momentum, which by Theorems $4.2,4.3$ is directly given by the spectrum of the time derivative of $F_{i, k}^{t}$ or of $c_{i, k}(t)$, at $t=0$.

\section{Examples}

The above abstract structures and theorems are illustrated by a large class of models [20].

i) General Spin and BCS Models. We consider the class of spin models defined by the standard spin algebra $\mathscr{A}$ on a lattice and the following finite volume 
Hamiltonians

$$
H_{V}=\frac{1}{|V|} \sum_{i, j \in V} \sum_{\alpha, \beta} \sigma_{\alpha}^{i} A_{\alpha \beta} \sigma_{\beta}^{j}+\sum_{i \in V} \sum_{\alpha} C_{\alpha} \sigma_{\alpha}^{i} .
$$

The choice $A_{\alpha \beta}=A \delta_{\alpha \beta}, C_{\alpha}=B \delta_{\alpha, 3}$ yields the Heisenberg model in the mean field approximation [21] with an external field $B$, whereas

gives the BCS model [13].

$$
A_{\alpha \beta}=-\frac{T_{c}}{2}\left(\begin{array}{rrr}
1 & i & 0 \\
-i & 1 & 0 \\
0 & 0 & 0
\end{array}\right), \quad C_{\alpha}=-\varepsilon \delta_{\alpha, 3}
$$

The finite volume dynamics $\alpha_{V}^{t}$ is defined by

where

$$
\begin{aligned}
\frac{d}{d t} \alpha_{V}^{t}\left(\sigma_{\delta}^{i}\right)= & -2 \varepsilon_{\alpha \delta \gamma}\left[A_{\alpha \beta} \alpha_{V}^{t}\left(\sigma_{\gamma}^{i}\right) \alpha_{V}^{t}\left(\sigma_{\beta}^{V}\right)+A_{\beta \alpha} \alpha_{V}^{t}\left(\sigma_{\beta}^{V}\right) \alpha_{V}^{t}\left(\sigma_{\gamma}^{i}\right)\right] \\
& -2 C_{\alpha} \varepsilon_{\alpha \delta \gamma} \alpha_{V}^{t}\left(\sigma_{\gamma}^{i}\right),
\end{aligned}
$$

$$
\sigma_{\alpha}^{V}=\frac{1}{|V|} \sum_{i \in V} \sigma_{\alpha}^{i}
$$

It is shown in ref. [20] that $\alpha_{V}^{t}\left(\sigma_{\delta}^{i}\right)$ converges ultrastrongly as $V \rightarrow \infty$, whenever $\sigma_{\alpha}^{V}$ converges ultrastrongly. An algebraic dynamics $\alpha^{t}$ is then defined by taking as family $F$ the largest set of states for which the ergodic limits (5.4) converge ultrastrongly. It is then shown in ref. [20] that the ultrastrong limit of $\alpha_{V}^{t}, \alpha^{t}$, defines a one parameter group of automorphisms of the von Neumann algebra $\mathfrak{M}$ defined as the weak closure of $\mathscr{A}$ with respect to $F$.

States $\phi$ invariant under time evolutions are easily constructed as product states with the property that

$$
\phi\left(\alpha^{t}\left(\sigma_{\alpha}^{\infty}\right)\right)=\phi\left(\sigma_{\alpha}^{\infty}\right), \quad \sigma_{\alpha}^{\infty}=\lim _{V \rightarrow \infty} \sigma_{\alpha}^{V} .
$$

For any representation $\pi$ defined by a pure state invariant under time translations the algebraic dynamics $\alpha^{t}$ gets effectively localized on the subalgebra $\mathscr{A}_{l}=\mathscr{A}$, (see Def. 3.1), and the corresponding automorphism $\alpha_{\pi}^{t}$ is defined as the solution of the following equation:

$$
\frac{d}{d t} \alpha_{\pi}^{t}\left(\sigma_{\delta}^{i}\right)=-2 \varepsilon_{\alpha \delta \gamma}\left(A_{\alpha \beta}+A_{\beta \alpha}\right) \pi\left(\sigma_{\beta}^{\infty}\right) \alpha_{\pi}^{t}\left(\sigma_{\gamma}^{i}\right)-2 C_{\alpha} \varepsilon_{\alpha \delta \gamma} \alpha_{\pi}^{t}\left(\sigma_{\gamma}^{i}\right) .
$$

For the proof of the above statements see ref. [20].

For simplicity we discuss the symmetry breaking aspects for the Heisenberg and the BCS models. For the Heisenberg model with $B=0$, the three dimensional spin rotations define automorphisms of $\mathscr{A}$ which commute with $\alpha_{V}^{t}$. Therefore, they can be extended to automorphisms of $\mathfrak{M}$ which commute with $\alpha^{t}$. Given a pure product state invariant under space translations $\phi_{0}^{\vec{n}}$, with $\phi_{0}^{\vec{n}}\left(\sigma_{\alpha}^{i}\right)=n_{\alpha}$, $|\vec{n}|=1, \phi_{0}^{\vec{n}}$ is time translation invariant and only the rotations around $\vec{n}$ are unbroken in the representation defined by $\phi_{0}^{\vec{n}}$.

The set $S$ (see Def. 4.2) is the set of states $\phi_{0}^{\vec{m}} ; Z_{S}$ is generated by $\sigma_{\alpha}^{\infty}$ and the 
classical motion at infinity defined by $\alpha_{\pi}^{t}$ on $Z_{S}$ is the group of rotations of $\sigma_{\alpha}^{\infty}$ around $\vec{n}=\pi\left(\vec{\sigma}^{\infty}\right)$, with frequency $\omega=4 A$. The products of $\alpha_{\pi}^{t}$ and the rotations around the two axes $\vec{e}_{1}, \vec{e}_{2}$ orthogonal to $\vec{n}$, generate the group of three dimensional rotations (condition $\beta^{\prime}$ )); the independent charges of condition $\beta$ ) are the generators of rotations around $\vec{e}_{1}$ and $\vec{e}_{2}$.

For the BCS model the rotations around the $z$ axis are automorphisms $\beta^{\lambda}$ of $\mathscr{A}$ which commute with $\alpha_{V}^{t}$. They are therefore symmetries of the infinite volume algebraic dynamics. Pure product states invariant under space translations $\phi_{0}^{\vec{n}}$, with $\phi_{0}^{\vec{n}}\left(\sigma_{\alpha}^{i}\right)=n_{\alpha}$ are time translation invariant if either $n_{\alpha}=(0,0, \pm 1)$ or $n_{\alpha}=\left(n_{1}, n_{2}, \varepsilon / T_{c}\right)$. We consider the second case in which $\beta^{\lambda}$ is broken. $\alpha_{\pi}^{t}$ defines rotations around $-\vec{n}$, with frequency $\omega=2 T_{c}$. We see here an explicit example of an interesting phenomenon which accompanies the symmetry breaking in the presence of long range interactions, namely the generation of exact symmetries of the effective dynamics $\alpha_{\pi}^{t}$ (here rotations around $\vec{n}$ ) which did not exist as symmetries of the finite volume Hamiltonians nor of the infinite volume dynamics $\alpha^{t}$.

The set $S$ is again the set of states $\phi_{0}^{\vec{m}},|\vec{m}|=1, Z_{S}$ is generated by $\sigma_{\alpha}^{\infty}$ and the classical motion at infinity is the group of rotations of $\sigma_{\alpha}^{\infty}$ around $\vec{n}=\pi\left(\vec{\sigma}^{\infty}\right)$, with frequency $\omega=2 T_{c}$. All the other features are very similar to the Heisenberg model, in particular the independent charges of condition $\beta$ ) may be taken as the generators of rotations around $z$ and around $\left(\vec{n} \cdot \vec{e}_{z}\right) \vec{n}+\vec{n} \times \vec{e}_{z}$.

For more details see ref. [20].

ii) Kibble Model. The abelian Higgs phenomenon is well illustrated by freezing the modulus of the scalar field in the Higgs-Kibble model. The dynamics of such a linearized model in the Coulomb gauge essentially reduces to that of a scalar field with Hamiltonian

$$
H=\frac{1}{2} \int d^{3} x\left[\pi^{2}+(\vec{\nabla} \varphi)^{2}\right]+\frac{1}{2} \int d^{3} x d^{3} y \pi(\vec{x}) U(\vec{x}-\vec{y}) \pi(\vec{y}), \quad U(\vec{x})=g^{2} /|\vec{x}|
$$

(Kibble model [5]). A proper discussion of the model requires an infrared regularization; we will introduce an infrared cutoff $L$ in the potential, e.g. $U_{L}(\vec{x})=$ $U(\vec{x}) f(|\vec{x}| / L)$, with $f$ a regular function which is one inside the sphere of radius 1 and vanishes outside the sphere of radius $1+a$. (The actual form of the infrared regularization is not important.)

The canonical commutation relations (CCR)

$$
[\varphi(\vec{x}), \pi(\vec{y})]=i \delta(\vec{x}-\vec{y})
$$

uniquely determine (see e.g. ref. [18] vol. II) an abstract $C^{*}$-algebra, generated by the Weyl operators $W(f), f=\left(f_{1}, f_{2}\right), f_{1}, f_{2} \in \mathscr{S}_{\text {real }}\left(R^{3}\right)$, which we will take as algebra $\mathscr{A}$.

The (formal) equations of motion given by the infrared cutoff Hamiltonian $H_{L}$ are

$$
\dot{\varphi}(\vec{x}, t)=\pi(\vec{x}, t)+\int U_{L}(\vec{x}-\vec{y}) \pi(\vec{y}, t) d^{3} y, \quad \dot{\pi}(\vec{x}, t)=\Delta \varphi(\vec{x}, t),
$$

which imply

$$
\ddot{\varphi}(\vec{x}, t)=\Delta \varphi(\vec{x}, t)-4 \pi g^{2} \varphi(\vec{x}, t)+\int \sigma_{L}(\vec{x}-\vec{y}) \varphi(\vec{y}, t) d^{3} y,
$$


where $\sigma_{L}$ has support in $L \leqq|\vec{x}-\vec{y}| \leqq L(1+a)$ and $\int \sigma_{L}(\vec{x}) d^{3} x=4 \pi g^{2}$. The $\sigma_{L}$ term is a consequence of the (necessary) infrared regularization, and it gives rise to infinitely delocalized variables in the limit $L \rightarrow \infty$.

For a rigorous construction of $\alpha^{t}$ we consider the infrared cutoff dynamics $\alpha_{L}^{t}$ on $\mathscr{A}: \alpha_{L}^{t} W(f)=W\left(f^{t}\right)$ with

$$
\begin{aligned}
\tilde{f}_{1}^{t}(\vec{k}) & =\cos \left(\omega_{L}(k) t\right) \tilde{f}_{1}(\vec{k})-\sin \left(\omega_{L}(k) t\right)\left(\vec{k}^{2} / \omega_{L}(k)\right) \tilde{f}_{2}(\vec{k}), \\
\tilde{f}_{2}^{t}(k) & =\sin \left(\omega_{L}(k) t\right)\left[\left(1+\tilde{U}_{L}(k)\right) / \omega_{L}(k)\right] \tilde{f}_{1}(k)+\cos \left(\omega_{L}(k) t\right) \tilde{f}_{2}(\vec{k}),
\end{aligned}
$$

where $\omega_{L}^{2}(k)=\vec{k}^{2}\left(1+\tilde{U}_{L}(k)\right)$.

The dynamics $\alpha_{L}^{t}$ commutes with the automorphism $\beta^{\lambda}$ of $\mathscr{A}$ defined by $\varphi \rightarrow \varphi+\lambda$, i.e.

$$
\beta^{\lambda} W(f)=\exp \left(i \lambda \tilde{f}_{1}(0)\right) W(f) .
$$

The states of $F$ can be taken as follows: one considers the set $F_{0}$ of Fock representations of $\mathscr{A}$ defined by translationally invariant states $\Omega$ with

$$
\Omega(W(f))=\exp \left[-\frac{1}{4}[f, f]\right],
$$

where

$$
[f, f]=\int d^{3} k \bar{f}_{i}(k) M_{i j}(k) f_{j}(k), \quad \bar{M}_{i j}(k)=M_{i j}(k)=M_{j i}(k), \quad \operatorname{det} M=1,
$$

and $M$ satisfies the following infrared regularity condition

$$
\left|M_{11}(k)\right| \leqq|\vec{k}|^{-3+\delta}, \quad\left|M_{22}(k)\right| \leqq|\vec{k}|^{1+\delta}, \quad \delta>0 .
$$

The set of states associated to the representations of $F_{0}$ and to their transform under $\beta^{\lambda *}, \lambda \in \mathbb{R}$, is then taken as family $F$. One can prove [20] that the infrared cutoff dynamics $\alpha_{L}^{t}(A), A \in \mathscr{A}$, converges ultrastrongly, with respect to $F$, as $L \rightarrow \infty$. In this way one defines a mapping $\alpha^{t}$ of $\mathscr{A}$ in $\mathfrak{M}$; one checks that $\alpha^{t^{*}} F \subset F$ and that $\alpha^{t_{1}{ }^{*}} \alpha^{t_{2}{ }^{*}}=\alpha^{\left(t_{1}+t_{2}\right)^{*}}$, and this implies that $\alpha^{t}$ defines a one parameter group of automorphisms of $\mathfrak{M}$, which commute with $\beta^{\lambda}$ [20].

The state $\phi_{0}$ of the form (5.13) with $M_{12}=0, M_{11}=\omega_{\infty}(k) / \vec{k}^{2}, M_{22}=\vec{k}^{2} / \omega_{\infty}(k)$ and its transforms under $\beta^{\lambda^{*}}$ are invariant under time translations. Each of them $\left(\phi_{\lambda}, \lambda \in \mathbb{R}\right)$ leads to an effective localization of the dynamics on the subalgebra $\mathscr{A}_{l} \subset \mathfrak{M}$ of finite linear combinations of the (extended) Weyl operators $W(f)$, with $f_{1}(\vec{x}) \in \mathscr{S}\left(R^{3}\right), \Delta f_{2}(\vec{x}) \in \mathscr{S}\left(R^{3}\right)$; the corresponding relocalized dynamics are given by

$$
\alpha_{\pi_{\lambda}}^{t}(W(f))=W\left(f^{t}\right) \exp \left[i \lambda \widetilde{f}_{1}(0)(1-\cos \omega t)+i \lambda\left(\vec{k}^{2} \widetilde{f}_{2}\right)(0) \sin \omega t / \omega\right],
$$

where $\omega=\omega_{\infty}(0)$ and $f^{t}$ is defined by Eq. (5.11) with $L=\infty$. In each representation defined by $\phi_{\lambda}, \beta^{\lambda}$ is broken.

The algebra $\mathscr{A}_{l}$ is stable under $\beta^{\lambda}$ and on $\mathscr{A}_{l}$,

$$
\left.\frac{d}{d \lambda} \phi_{0}\left(\beta^{\lambda}(A)\right)\right|_{\lambda=0}=i \lim _{R \rightarrow \infty} \phi_{0}\left(\left[Q_{R}, A\right]\right), \quad A \in \mathscr{A}_{l},
$$

where

$$
Q_{R}=\int \pi(\vec{x}) f_{R}(\vec{x}) d^{3} x
$$


One checks that the charge is integrable as a commutator for $A \in \mathscr{A}_{l}$ (Eq. (4.4)).

Furthermore the mapping

$$
\gamma^{\lambda}(W(f))=W(f) \exp \left[i \lambda\left(\vec{k}^{2} \widetilde{f}_{2}\right)(0)\right]
$$

defines a one parameter group of automorphisms of $\mathscr{A}_{l}$ and

$$
\begin{aligned}
& \beta^{\lambda} \alpha_{\pi}^{t}=\alpha_{\pi}^{t} \beta^{\lambda \cos \omega t} \gamma^{-\lambda \sin \omega t / \omega}, \\
& \beta^{\lambda} \gamma^{\lambda^{\prime}}=\gamma^{\lambda^{\prime}} \beta^{\lambda}
\end{aligned}
$$

(conditions $\beta$ and $\beta^{\prime}$ are satisfied).

The set $S$ is the set of states $\beta^{\lambda^{*}} \gamma^{\mu^{*}} \phi_{0} \equiv \phi^{\lambda, \mu}$ and

$$
\alpha_{\pi}^{t^{*}} \phi^{\lambda, \mu}=\phi^{\lambda \cos \omega t+\mu \omega \sin \omega t, \mu \cos \omega t-\lambda \sin \omega t / \omega} .
$$

$Z_{S}$ is generated by

$$
\begin{aligned}
\exp \left[i a \varphi_{\infty}\right] & =w-\lim _{L \rightarrow \infty} \exp \left[i a \varphi\left(L^{-3} h(|\vec{x}| / L)\right],\right. \\
\exp \left[i a\left(\frac{1}{4 \pi r} * \pi\right)_{\infty}\right] & =w-\lim _{L \rightarrow \infty} \exp \left[i a\left(\frac{1}{4 \pi r} * \pi\right)\left(L^{-3} h(|\vec{x}| / L)\right)\right],
\end{aligned}
$$

where $\int h(\vec{x}) d^{3} x=1$, and the classical dynamics at infinity can be written as

$$
\alpha_{\pi}^{t} \varphi_{\infty}=\varphi_{\infty} \cos \omega t+\left(\frac{1}{4 \pi r} * \pi\right)_{\infty} \sin \omega t / \omega .
$$

The energy gap associated to the breaking of $\beta^{\lambda}$ is $\omega$.

The standard treatment of this model [5] completely misses the following essential features: i) the correct treatment of the dynamics requires us to define it as a limit of infrared cutoff time evolutions; ii) this procedure accounts for the symmetry of the algebraic dynamics under $\beta^{\lambda}$ through the appearance of variables at infinity, which are not present in the Hamiltonian; this feature is due to the interplay between the kinetic term and the long range $1 / r$ of the interaction; iii) the effective localization of the dynamics is obtained by freezing the variables at infinity to their expectation values and this mechanism explains the generation of energy gap.

The same features show up also in the Kogut-Susskind model [8] if correctly treated along the above lines [20].

\section{References}

This is a shortened version of a paper with the same title (ISAS report 35/84/E.P.) hereafter referred as [I].

1. Goldstone, J., Salam, A., Weinberg, S.: Broken symmetries. Phys. Rev. 127, 965 (1962)

2. Kastler, D., Robinson, D. W., Swieca, A.: Conserved currents and associated symmetries: Goldstone's theorem. Commun. Math. Phys. 2,108 (1966); Ezawa, H., Swieca, J. A.: Spontaneous breakdown of symmetries and zero-mass states. Commun. Math. Phys. 5, 330 (1967); Swieca, J.: Goldstone theorem and related topics. In: Cargèse Lectures in Physics. Vol. 4, D. Kastler, (ed.), New York: Gordon and Breach 1970

3. Coleman, S.: Secret symmetries, Erice Summer School 1973; Zichichi, A. (ed.) by Academic Press 1975, New York: distributed 
4. Haag, R.: The mathematical structure of the BCS model. Nuovo Cimento 25, 281 (1962)

5. Kibble, T. W. B.: Proc. intern. conf. elementary particles, p. 19, Oxford: Oxford Univ. Press 1965

6. Fröhlich, J., Morchio, G., Strocchi, F.: Higgs phenomenon without a symmetry breaking order parameter. Phys. Letters 97B, 249 (1980), Nucl. Phys. B190, [FS3], 553 (1981)

7. Weinberg, S.: The $U(1)$ problem. Phys. Rev. D11, 3583 (1975); Crewther, R. J.: Chiral properties of quantum chromodynamics. In: Field theoretical methods in particle physics. W. Rühl, ed., New York: Plenum Press 1980

8. Kogut, J., Susskind, L.: How quark confinement solves the $\eta \rightarrow 3 \pi$ problem. Phys. Rev. D11, 3594 (1975)

9. Lanford, O. E., III, Ruelle, D.: Observables at infinity and states with short range correlations in statisticals mechanics. Commun. Math. Phys. 13, 194 (1969)

10. Strocchi, F.: Gauss' law in local quantum field theory. In: Field theory, quantization and statistical physics. E. Tirapegni, (ed.) Amsterdam: D. Reidel 1981

11. Haag, R., Hugenholz, N. M., Winnink, M.: On the equilibrium states in quantum statistical mechanics. Commun. Math. Phys. 5, 215 (1967); Robinson, D. W.: Statistical mechanics of quantum spin systems. II. Commun. Math. Phys. 7, 337 (1968)

12. Dubin, D. A., Sewell, G. L.: Time translations in the algebraic formulation of statistical mechanics. J. Math. Phys. 11, 2990 (1970); Ruskai, M. B.: Time development of quantum lattice systems. Commum. Math. Phys. 20, 193 (1971); Sewell, G. L.: States and dynamics of infinitely extended physical systems. Commun. Math. Phys. 33, 43 (1973)

13. Thirring, W.: On the mathematical structure of the B.C.S.-model. II. Commun. Math. Phys. 7, 181 (1968); Lectures at the international school of physics, Mallorca 1968, New York: Plenum Press

14. Hagg, R., Kadison, R. V., Kastler, D.: Nets of $C^{*}$-algebras and classification of states. Commun. Math. Phys. 16, 81 (1970)

15. Kadison, R. V.: Topology 3, 177 (1965)

16. Swieca, J. A.: Range of forces and broken symmetries in many-body systems. Commun. Math. Phys. 4, 1 (1967)

17. Swieca, J. A.: Goldstone's theorem and related topics. In: Cargèse Lectures. Vol. 4, New York: Gordon and Breach, 1970

18. Brattelli, O., Robinson, D. W.: Operator algebras and quantum statistical mechanics. I, pp. 396-397, Berlin-Heidelberg-New York: Springer 1979

19. Morchio, G., Strocchi, F.: Spontaneous breaking of the Galilei group and the plasmon energy gap, IFUP TH $85 / 8$

20. Morchio, G., Strocchi, F.: Long range dynamics and broken symmetries. I. Spin models; II. Gauge models (in preparation)

21. Hugenholtz, N. M.: Equilibrium States in Stat. Mech. In: Lectures in Theor. Phys. Vol. XIV B, Boulder 1971, W. E. Brittin (ed.), Boulder: Colorado Univ. Press 1973

Communicated by R. Haag

Received June 18, 1984; shortened version October 14, 1984 
